\title{
Evolution of endogenous retroviruses in the Suidae: evidence for different viral subpopulations in African and Eurasian host species
}

\author{
Fabrícia F Nascimento ${ }^{1,5}$, Jaime Gongora' ${ }^{1}$, Michael Charleston, Michael Tristem ${ }^{3}$, Stewart Lowden ${ }^{4}$ and \\ Chris Moran ${ }^{1 *}$
}

\begin{abstract}
Background: Porcine endogenous retroviruses (PERVs) represent remnants of an exogenous form that have become integrated in the domestic pig (Sus scrofa) genome. Although they are usually inactive, the capacity of $\gamma 1$ ERVs to infect human cells in vitro has raised concerns about xenotransplantation because the viruses could cross the species barrier to humans. Here we have analyzed the evolution of $\gamma 1$ ERVs in ten species of Suidae (suids, pigs and hogs) from Eurasia and Africa using DNA sequences for their coding domains (gag, pro/pol and env genes). For comparison with $\gamma 1$ PERVs, we have also analysed $\gamma 2$ ERVs which in domestic pigs are known to be inactive and do not pose a risk to xenotransplantation.

Results: Phylogenetic analysis using Bayesian inference showed that $\gamma 1$ and $\gamma 2$ ERVs have distinctive evolutionary histories. Firstly, two different viral lineages of $\gamma 1$ ERVs were found and a coevolutionary analysis demonstrated that they correspond broadly to their host phylogeny, one of Eurasian and another of African species, and show no evidence of horizontal transmission. $\gamma 2$ ERVs, however, show a bush-like evolution, suggesting a rapid viral radiation from a single common ancestor with no correspondence between host and viral evolutionary trees. Furthermore, though $\gamma 1$ ERV env genes do not possess frequent stop codons, $\gamma 2$ env genes do. To understand whether $\gamma 1$ suid ERVs may be still replicating, we have also evaluated their likely mechanism of proliferation by statistically testing internal to terminal branches using nonsynonymous versus synonymous substitution ratios. Our results suggest that $\gamma 1$ ERVs are increasing in copy number by reinfection, which requires the translocation of the virus from one cell to another.

Conclusions: Evidence of at least two viral subpopulations was observed in $\gamma 1$ ERVs from Eurasian and African host species. These results should be taken into account in xenotransplantation since $\gamma 1$ ERVs appear to be codiverging with their host and maintaining ongoing capacity to infect somatic and germ cells.
\end{abstract}

\section{Background}

Endogenous retroviruses (ERVs) are the remnants of an exogenous viral form that infected and became integrated in germ cell genomes [1]. ERVs have been found in a variety of vertebrates, including reptiles, amphibian, birds and mammals [2]. However, the occurrence and evolution of ERVs in different organisms are still not well understood and they are sometimes lumped with "junk" DNA of unknown function [3,4].

\footnotetext{
* Correspondence: christopher.moran@sydney.edu.au

${ }^{1}$ Faculty of Veterinary Science, The University of Sydney, NSW 2006, Australia Full list of author information is available at the end of the article
}

In the domestic pig and wild boar (Sus scrofa) genome, ERVs are referred to as PERVs (porcine endogenous retroviruses) and belong to the $\beta$ and $\gamma$ genera comprising several viral groups $[5,6]$. The $\gamma 1$ genus is divided in PERV-A, B and C classes on the basis of env sequences, while the $\gamma 2$ genus comprises only PERV-E. Although most PERVs carry mutations that render them functionally inactive, some $\gamma 1$ PERVs retain the ability to express and can infect human cells in vitro [7,8]. The capacity of $\gamma 1$ ERVs to infect human cells in tissue culture raised serious concerns on the safety of xenotransplantations $[9,10]$ because pigs are the first choice of xenograft donors

\section{Ciomed Central}


[11-13]; consequently $\gamma 1$ ERVs have been extensively studied in Sus scrofa.

In contrast, few DNA sequences from $\gamma 2$ ERVs have been reported to date $[5,14,15]$, although a full length provirus has been described and named PERV-E because of its similarity to the human HERV-E. Furthermore, PERV-E forms a distinct clade with viruses from the HERV-E family and does not cluster with $\gamma 1$ PERVs [14]. Genetic and expression analyses also have not indicated an obvious infectious risk from $\gamma 2$ retroviruses for xenotransplantation [15].

At present, little is known about either $\gamma 1$ or $\gamma 2$ ERVs in other suid species than Sus scrofa $[5,16]$. Patience et al. [5] screened by PCR for the presence of ERVs (both $\gamma$ and $\beta$ genera) in five different suid species, while Niebert and Tönjes [16] sequenced $\gamma 1$ DNA fragments in seven different suid species. However, they did not perform any evolutionary analyses of ERVs [16]. Clearly both of these studies have left many questions unanswered.

The mechanisms by which $\gamma 1$ ERVs increase their copy number in suid genomes are also poorly understood and three main mechanisms have been reported for human ERVs (HERVs) [17,18]; retrotransposition in cis, complementation in trans, and reinfection [19]. The first mechanism involves replication by co-packaging mRNAs in virus-like particles built up from viral proteins encoded by their own gag and pol genes. As retrotransposition in cis does not result in cell to cell translocation, the env gene is not required to be functional. Conversely, complementation in trans involves replication of two retroviral elements of the same family, with one defective provirus using the functional protein produced by another provirus. Finally, ERVs might form infectious exogenous particles and reinfect either a germ cell or a somatic cell. Because reinfection requires the translocation of viruses from one cell to another, the presence of functional genes is required, although this may also result from complementation in trans $[17,20]$. New ERVs will likely acquire new mutations during reinfection or retrotransposition due to their error prone reverse transcriptase in virus replication [19]. Thus proviruses might occasionally give rise to infectious exogenous forms which can reinfect the animal itself or animals of the same species and sometimes cross to other species, and subsequently integrate in a germ cell [21].

Recently a study of the host phylogeny [22,23] clarified the relationship between Eurasian and African suids based on nuclear and mitochondrial DNA sequences, enabling the study of ERV evolution in these suid species and the evolutionary congruence of host and viral lineages. Here, we analyzed the $\gamma 1$ ERV coding domains (gag, pro/pol and env) sampled from 10 different suid host species from Eurasia and Africa. We have analyzed their evolutionary relationships as well as their likely mechanism of replication. We have also compared the evolution of $\gamma 1$ and $\gamma 2$ ERVs using their env genes and implication for xenotransplantation.

\section{Results}

\section{Screening of ERV sequences}

A total of 144 partial $\gamma 1$ ERV sequences comprising the pol, gag and env (classes A, B and C) genes and $40 \gamma 2$ env were generated (Table 1). One individual for each suid species was analyzed, except for the babirusa, where two animals were analyzed for env class E. No $\gamma 1$ ERV genes were amplified in the babirusa, while pol and env class A and $\mathrm{C}$ were not amplified in the forest hog, and pol and env class A were not amplified in the Javan warty pig and the desert warthog, respectively. We were able to amplify $\gamma 2$ ERVs in all of the species.

Blastn confirmed that amplicons corresponded to ERVs. Primers targeting env classes A and C cross amplified. Sequences generated with these primers were initially analyzed together and then separated into A and $C$ according to blastn results. After reorganization, a total of 18 and 22 sequences for class $\mathrm{A}$ and $\mathrm{C} e n v$ were analyzed. env class $\mathrm{C}$ was not amplified from any African specimens, obviating the need for the non-parametric test for panmixia for this gene.

Three $g a g$ and one env class E sequences showed indels longer than $50 \mathrm{bp}$ and were removed from alignments to increase accuracy of evolutionary analysis. This allowed a higher number of base pairs to be analyzed because these indels were observed in different positions. After removing these sequences, a total of 141 novel $\gamma 1$ sequences were analyzed along with 28 sequences from the draft pig genome and 110 sequences from GenBank (Additional file 1). These sequences showed a pairwise genetic distances in expected number of substitutions per site ranging from $0-0.10$ for pol, $0-0.11$ for $g a g, 0-0.17$ for env class A, $0-0.27$ for env class B, and $0-0.15$ for $e n v$ class $C$. Identical sequences were rarely observed, and they mainly reflect identical sequences from GenBank and between Genbank and Sus scrofa genome sequences. Non-parametric tests revealed highly significant differentiation between ERVs from African versus Eurasian species $(P<0.001)$. On the other hand, no sequence was removed from $\gamma 2$ env dataset for which pairwise genetic distances ranged from $0.001-0.11$. The non-parametric test of population subdivision suggested that Eurasian, African and babirusa sequences came from different subpopulations $(P<0.02)$.

In addition, most DNA sequences of $\gamma 1$ ERV genes lacked stop codons or, when present, were limited to one stop codon per sequence. This contrasted with $\gamma 2$ env, which possessed several stop codons (up to 5 per sequence) and the presence of several indels, some of which were in unique positions and others at conserved 
Table 1 List of species of Suidae showing their origin and number of sequences generated

\begin{tabular}{|c|c|c|c|c|c|c|c|c|c|c|}
\hline Origin & Species and subspecies & Common name & Source & Tissue & gag & pol & env $\mathrm{A}^{+}$ & env B & env $\mathrm{C}^{+}$ & env $\mathrm{E}$ \\
\hline \multirow[t]{5}{*}{ Africa } & Hylochoerus meinertzhageni & Forest hog & Uganda & Muscle & 3 & - & 1 & 3 & - & 3 \\
\hline & Phacochoerus aethiopicus & Desert warthog & Kenya & Muscle & 3 & 4 & - & 3 & - & 3 \\
\hline & Phacochoerus africanus & Common warthog & Iwaba Zimbabwe & Muscle & 7 & 7 & 6 & 1 & - & 4 \\
\hline & Potamochoerus larvatus & Bush-pig & Zimbabwe & Blood & 3 & 4 & 3 & 3 & - & 2 \\
\hline & Potamochoerus porcus & Red river hog & Duisburg Zoo, Germany & Muscle & 4 & 4 & 4 & 3 & - & 3 \\
\hline Eurasia & Sus scrofa & Wild boar & Yorkshire Farm, UK & Blood & 4 & 4 & 2 & 4 & 3 & 2 \\
\hline \multirow[t]{6}{*}{ Asia Pacific } & Sus barbatus oi & Western bearded pig & Singapore Zoo, Singapore & Blood & 4 & 4 & - & 3 & 4 & 3 \\
\hline & Sus barbatus barbatus & Bornean bearded pig & Singapore Zoo, Singapore & Blood & 4 & 3 & - & 4 & 6 & 2 \\
\hline & Sus celebensis & Sulawesi warty pig & Sulawesi, Indonesia & Muscle & 3 & 3 & 2 & 4 & 3 & 3 \\
\hline & Sus verrucosus & Javan warty pig & Poznan Zoo, Poland & Muscle & 3 & - & - & 5 & 6 & 4 \\
\hline & Babyrousa babyrussa & Babirusa & Surabaya Zoo, Indonesia & Blood & - & - & - & - & - & $11^{*}$ \\
\hline & & & & TOTAL & 38 & 33 & 18 & 33 & 22 & 40 \\
\hline
\end{tabular}

+ After reorganization of sequences (see text).

* Two animals were analyzed.

positions across Eurasian, African and babirusa sequences, suggesting that they are unlikely to be replicating as env is necessary for viral reinfection. Possibly, they might retrotranspose in cis if other coding domains were devoid of stop codons, although this is apparently unlikely because Sus scrofa gag and pro/pol also showed stop codons and frame shift mutations. Interestingly, there were three $\gamma 2$ sequences from African species (two from common warthog and one from desert warthog) and two from babirusa that did not show stop codons.

\section{Test for detecting recombination}

From $30 \%$ to $79 \%$ of sequences were identified as recombinants for $\gamma 1$ genes by the RDP 3 software [24] including some apparent inter-group recombinants (Figure 1). This program also identifies recombination breakpoints, which for our dataset were located at different positions when the aligned viral sequences are compared. Because of that, the intervening non-recombinant fragments of sequence were not useful for phylogenetic reconstructions because more than $50 \%$ of sequence length had to be removed from the alignment. Furthermore, in most cases, sequences identified as non-recombinant were not representative of the different suid species available in our dataset. We therefore did not use the non-recombinants identified by RDP 3 for phylogenetic reconstructions.

From $17 \%$ to $40 \%$ of sequences were identified as recombinants for $\gamma 1$ genes by the PHI-NNet algorithm [25], and a higher frequency was mainly observed in sequences from African suids but less frequently than with RDP 3 (Figure 1). Apparent inter-group recombinants between Eurasian and African sequences were identified only for env, albeit with a low frequency (Figures $1 \mathrm{C}$ and 1D). It is interesting to note that approximately $50 \%$ of env class A sequences in African suids appeared to be recombinants (Figure $1 \mathrm{C}$ ) while all env class $\mathrm{C}$ sequences, found only in Eurasian pigs, were likely to be recombinants and thus could not be used for evolutionary analyses. Because this algorithm identified a smaller number of recombinants, we used the observed non-recombinant dataset in our phylogenetic reconstructions.

Because approximately $50 \%$ of recombinants were observed for $\gamma 2$ env, with even some apparent intergroup recombinants detected by the PHI-NNet algorithm (Figure 2), the non-parametric test was re-run using only the non-recombinant dataset. In this case, the null hypothesis of panmixia could not be rejected $(P=0.07)$, providing no conclusive evidence that $\gamma 2$ ERVs from Eurasian and African species and babirusa represent different retroviral subpopulations.

\section{Phylogeny reconstructions}

In the Suidae, $\gamma 1$ ERVs are grouped into two different lineages, one in Eurasian and another in African suids, as indicated by class A and B env phylogenies (Figures 3 and 4). Furthermore, the pol phylogeny revealed five groups, four comprising only sequences from Eurasian species, and another mainly with African species in a well defined clade (Figure 5). However, one $\gamma 1$ pol sequence present in the draft pig genome grouped with $\gamma 1 \mathrm{pol}$ sequences of African suids. This sequence was identified as a recombinant by the RDP 3 software but not by the PHI-NNet algorithm.

The clades shown for African class A and B env phylogenies (Figures 3 and 4) were generally coincident with African host clades, except for the internal arrangement of Potamochoerus porcus and Hylochoerus meinertzhageni in the env class B phylogeny (Figure 4), which differed from the host tree [22,23] where Hylochoerus grouped with Phacochoerus instead of 


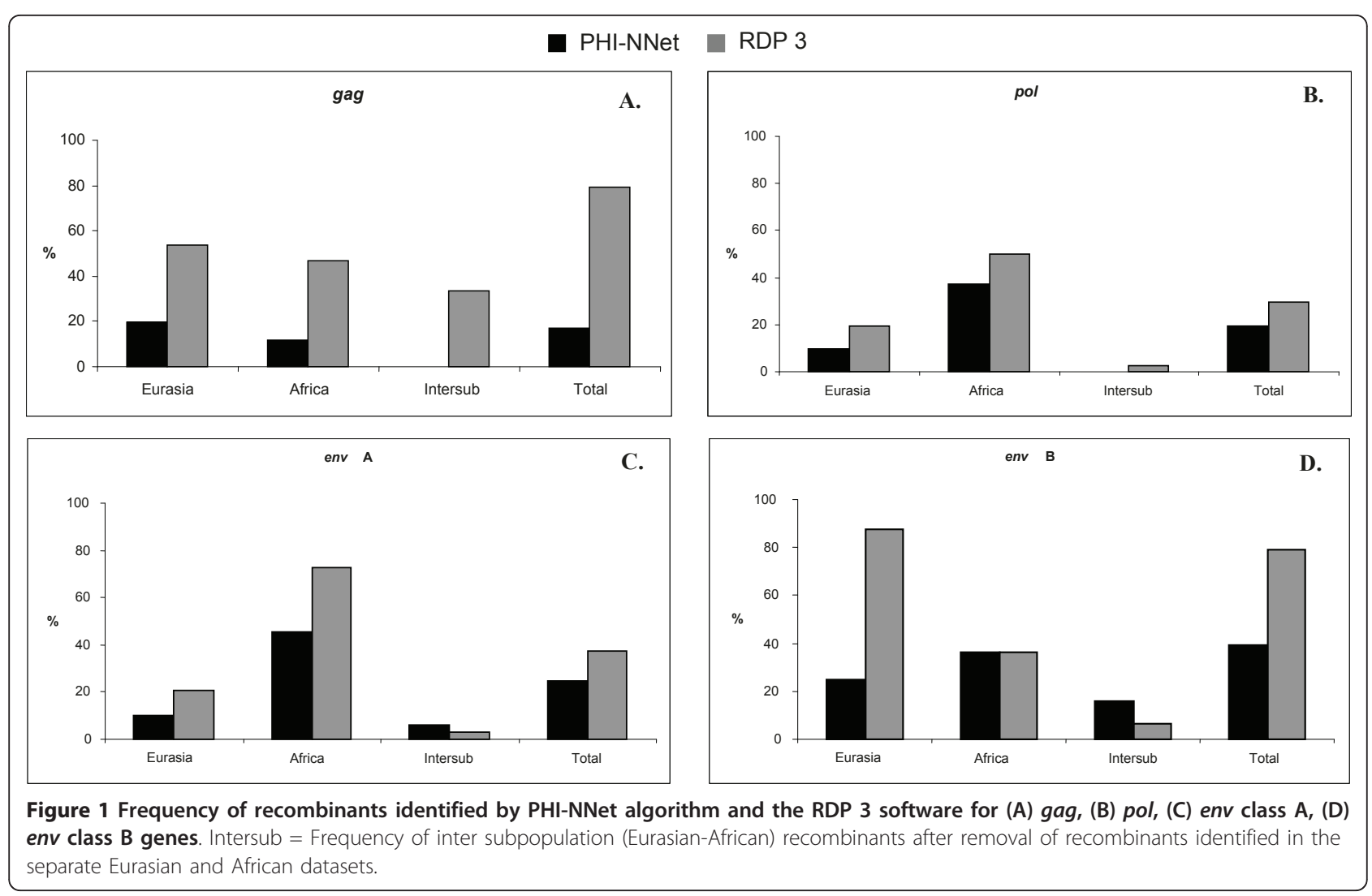

Potamochoerus. On the other hand, Eurasian $\gamma 1$ ERVs showed a less conserved arrangement in all phylogenies, with internal groups not corresponding to the host phylogeny [22,23] except for the env class B internal arrangement, which was very similar in the host phylogeny (highlighted in Figure 4).

The gag phylogeny did not group all African ERVs in a single clade, although most of them were still

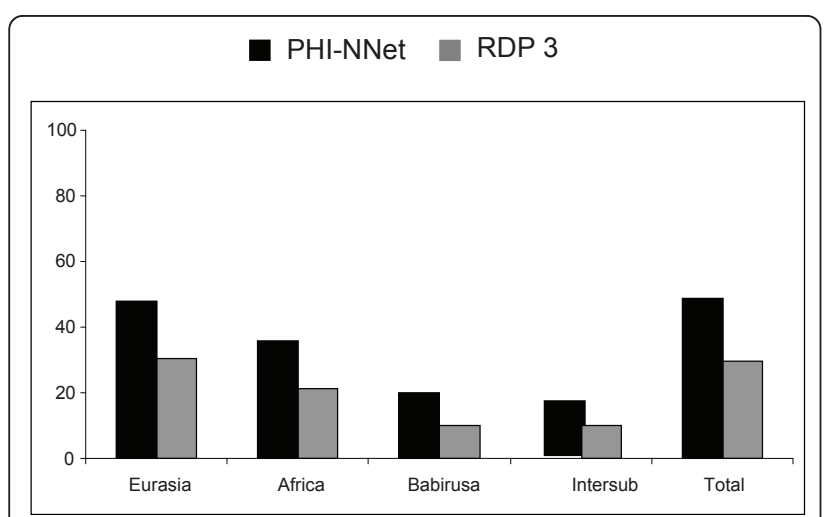

Figure 2 Frequency of recombinants identified by PHI-NNet algorithm and the RDP 3 software for env class E gene. Intersub = Frequency of inter subpopulation (Eurasian-African-Babirusa) recombinants after removal of recombinants identified in the separate Eurasian, African and Babirusa datasets. different from Eurasian ones (highlighted in Figure 6). A similar pattern is also observed when recombinant sequences detected by RDP 3 are also removed from phylogenetic analysis (data not shown). This suggests that recombination is not responsible for the lack of resolution on gag. Further conclusions based solely on gag should be carefully evaluated. Furthermore, these sequences did not show substitution saturation, with $\mathrm{I}_{\mathrm{ss}}$ much smaller than the critical $\mathrm{I}_{\mathrm{ss}}$ value $[26,27]$.

Conversely, Bayesian analysis for $\gamma 2$ suid ERVs showed a bush-like pattern (Figure 7) with short internal and long external branches. The same pattern was observed using neighbor-joining, and also with the complete sequence dataset (including recombinants), suggesting that for $\gamma 2$ ERVs, recombinants were not responsible for the star-like phylogeny. An unresolved phylogeny may also result from data saturation, although $\gamma 2$ env showed very low saturation, with $I_{\mathrm{ss}}=0.16$, below the critical $I_{\mathrm{ss}}$ value $\left(I_{\text {ss.c }}=0.77\right)[26,27]$. Similar results were obtained for first, second and third codon positions. Furthermore, the likelihood-mapping method indicated that the data contain a high amount of net-like (9.1\%) and star-like $(16.3 \%)$ signals, indicating that the tree was not well resolved according to the criteria of Strimmer and von Haeseler [28]. 


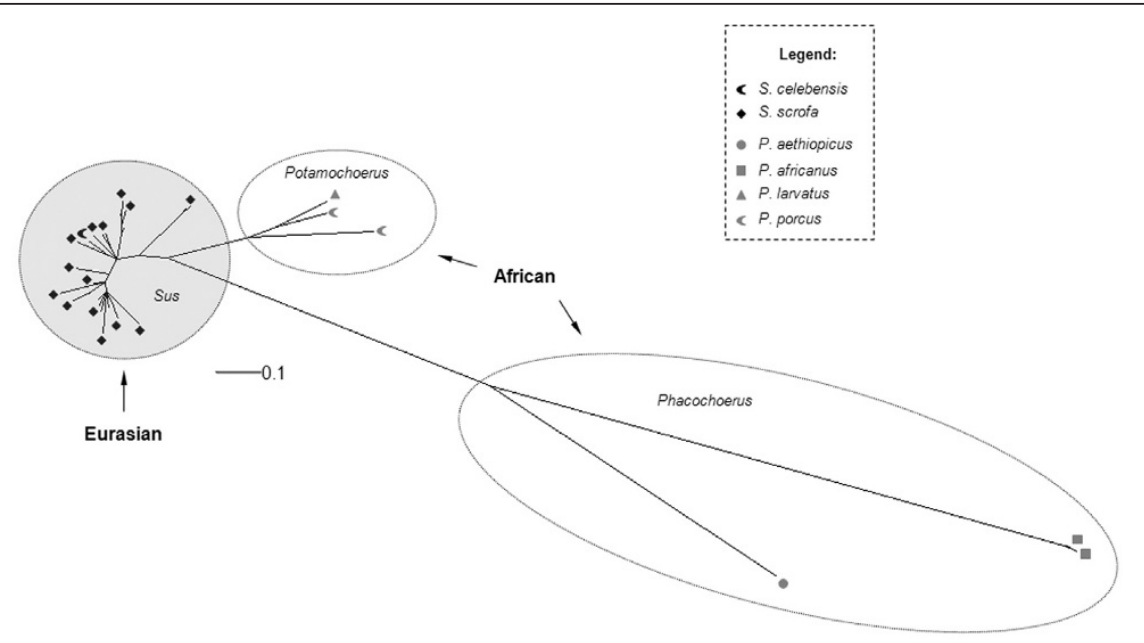

Figure 3 Unrooted Bayesian inference for env class A gene. For clarity, posterior probability values are not represented, but all main groups showed a value of 0.99 to 1.00. Black symbols represent ERVs from the different Eurasian species and gray symbols represent ERVs from different African species (see legend for detail in coding).

\section{Mechanism of ERV proliferation}

Most sequences of $\gamma 1$ ERVs did not possess stop codons and/or indels. Furthermore, when present, they were usually located at unique positions in any given sequence. Low frequency of stop codons is evidence that complementation in trans has been a rare event [18].

Estimates of $\omega$ for each branch in the phylogeny were used for testing the possibility of purifying selection $[29,30]$. Comparisons of internal branches with external branches ("two-ratio" model) suggested that purifying selection was acting on all genes because of the highly significant differences in log-likelihoods (Table 2).
Furthermore, $\omega$ was significantly smaller for internal branches than for external branches: 0.34 and 0.78 for gag, 0.25 and 0.56 for pol, 0.30 and 1.04 for env class A, and 0.22 and 1.47 for env class B (Table 2).

For the $\gamma 2$ env gene, we found statistical support for the "one-ratio" model $(P=0.053)$, which made testing the "two-ratio" model unnecessary.

\section{Discussion}

We found strong evidence that $\gamma 1$ ERVs from Eurasian and African species based on the pol and env phylogenies belong to two different lineages. This pattern was also observed for LTR phylogenies sampled from

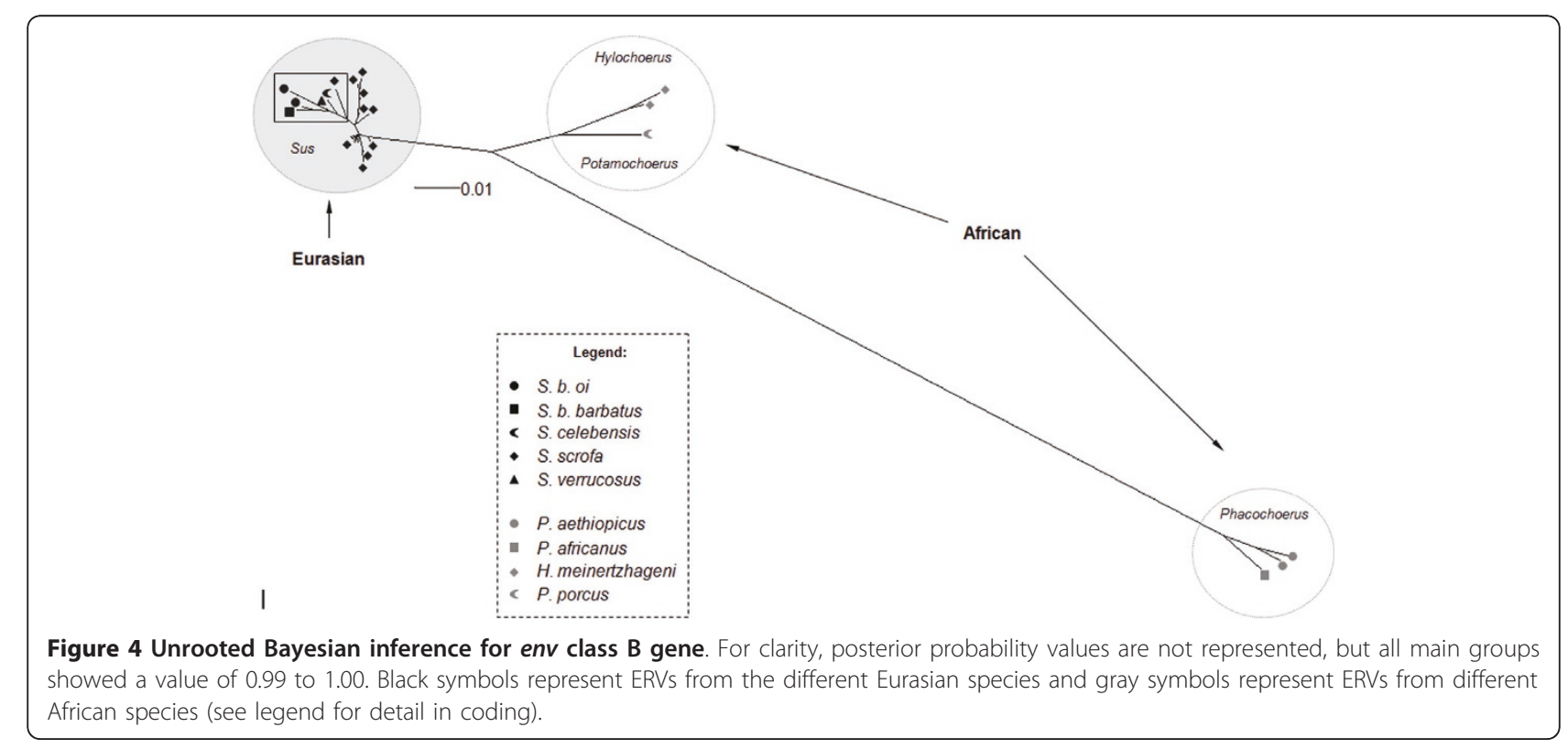




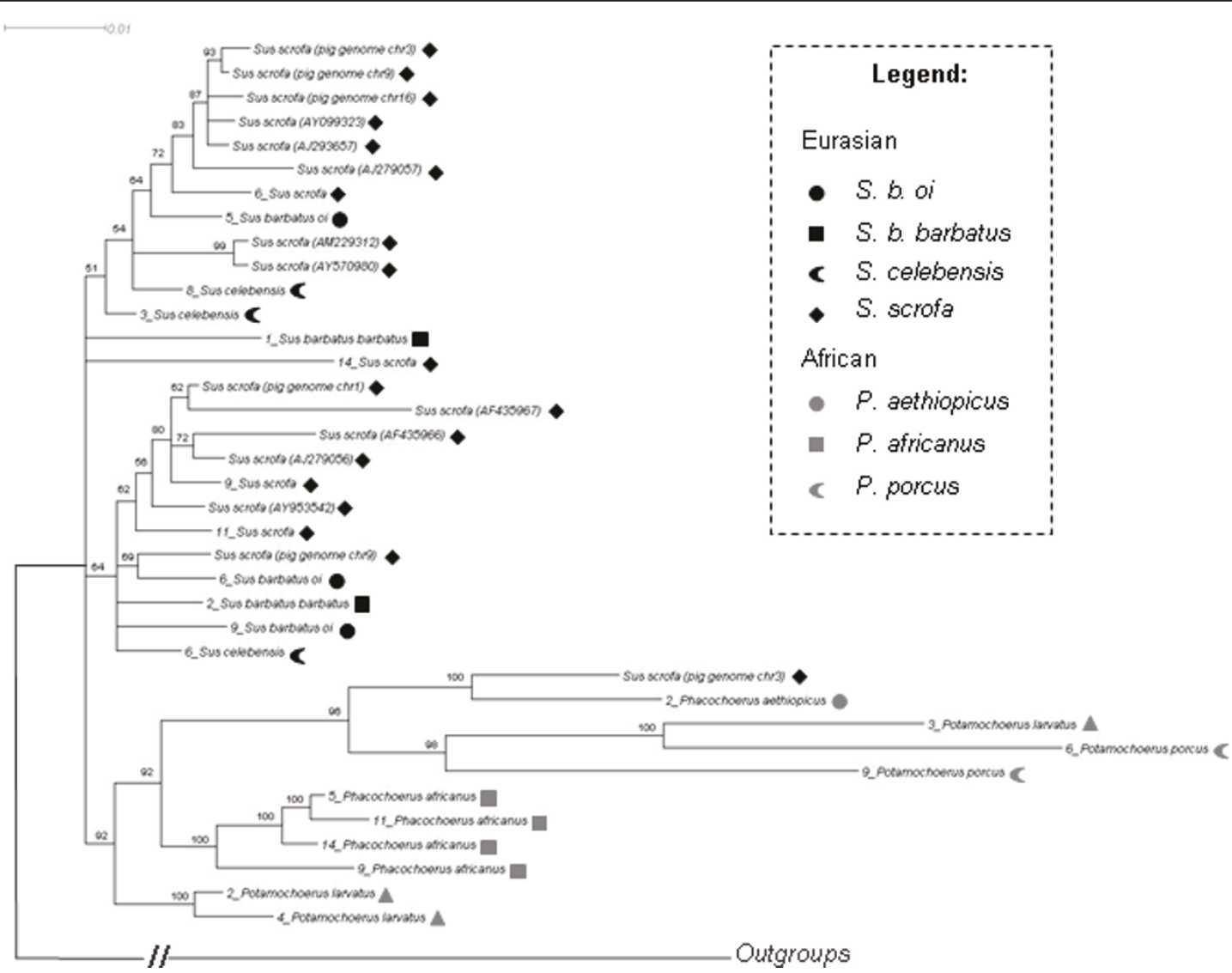

Figure 5 Rooted Bayesian inference for pol gene. Numbers close to branches indicate posterior probability values above 0.50. Numbers close to names correspond to different clones. Black symbols represent ERVs from the different Eurasian species and gray symbols represent ERVs from different African species (see legend for detail in coding). Outgroup branch is not to scale.

Eurasian and African host species [31]. Host species from Eurasia are more similar to one another, showing shorter branch lengths compared to the more differentiated African host species with longer branch lengths, consistent with traditional taxonomy which puts all Eurasian species in the genus Sus, whereas there are several genera recognized in Africa [32-34]. A similar pattern was generally observed in $\gamma 1$ ERV class A and B env phylogenies and for the LTRs, suggesting that proviruses might be codiverging with their respective host species.

The resolution of the pol phylogeny into distinct Eurasian and African lineages, which was not found with gag, is not a consequence of analysis of longer alignments. Bayesian analysis based on 885 bp of pol sequence (the same size as the gag alignment) showed an almost identical topology to that observed in Figure 5, showing a single African clade of ERVs (including the one ERV sequence of the draft pig genome). Similarly, when ca. 400 bp of env is analysed, an almost identical topology of that of Figure 3 is also observed [30].
The gag phylogeny is different from the other viral gene phylogenies and to the host phylogeny $[22,23]$. This may be explained by the gene tree versus species tree problem in which gene phylogenies sometimes conflict with a species tree because individual gene sequences can generate different topologies and consequently conflicting results [35-37], but is also likely to be due simply to the lack of resolution in the gag phylogeny which cannot be attributed to the length of base pairs analysed compared to the other genes. The lack of resolution of this gene was also observed by other authors when analysing other retroviruses [for an example see [38,39]]. Alternatively, the DNA sequence conservation of gag gene suggests that Gag proteins are important for viral replication. Experimental analysis of Moloney murine leukemia virus showed that mutations of several portions of $\mathrm{gag}$ were incompatible with viral replication [40], implying strong selection for conservation of the gag gene sequence. In this case, while other viral domains might have evolved distinct African and Eurasian sequences, gag might have been constrained from doing so. 


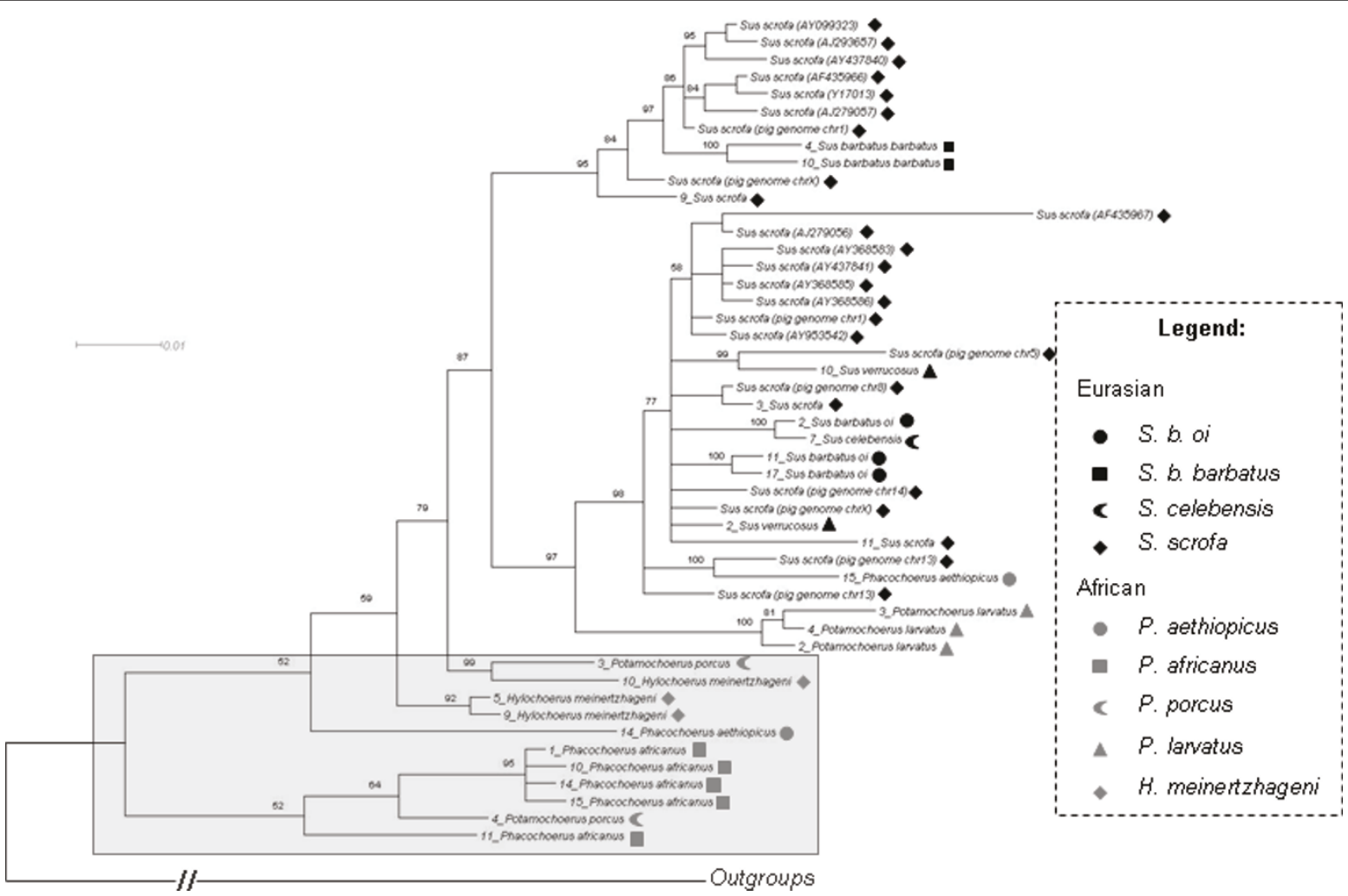

Figure 6 Rooted Bayesian inference for gag gene. Numbers close to branches indicate posterior probability values above 0.50. Numbers close to names correspond to different clones. Black symbols represent ERVs from the different Eurasian species and gray symbols represent ERVs from different African species (see legend for detail in coding). Outgroup branch is not to scale.

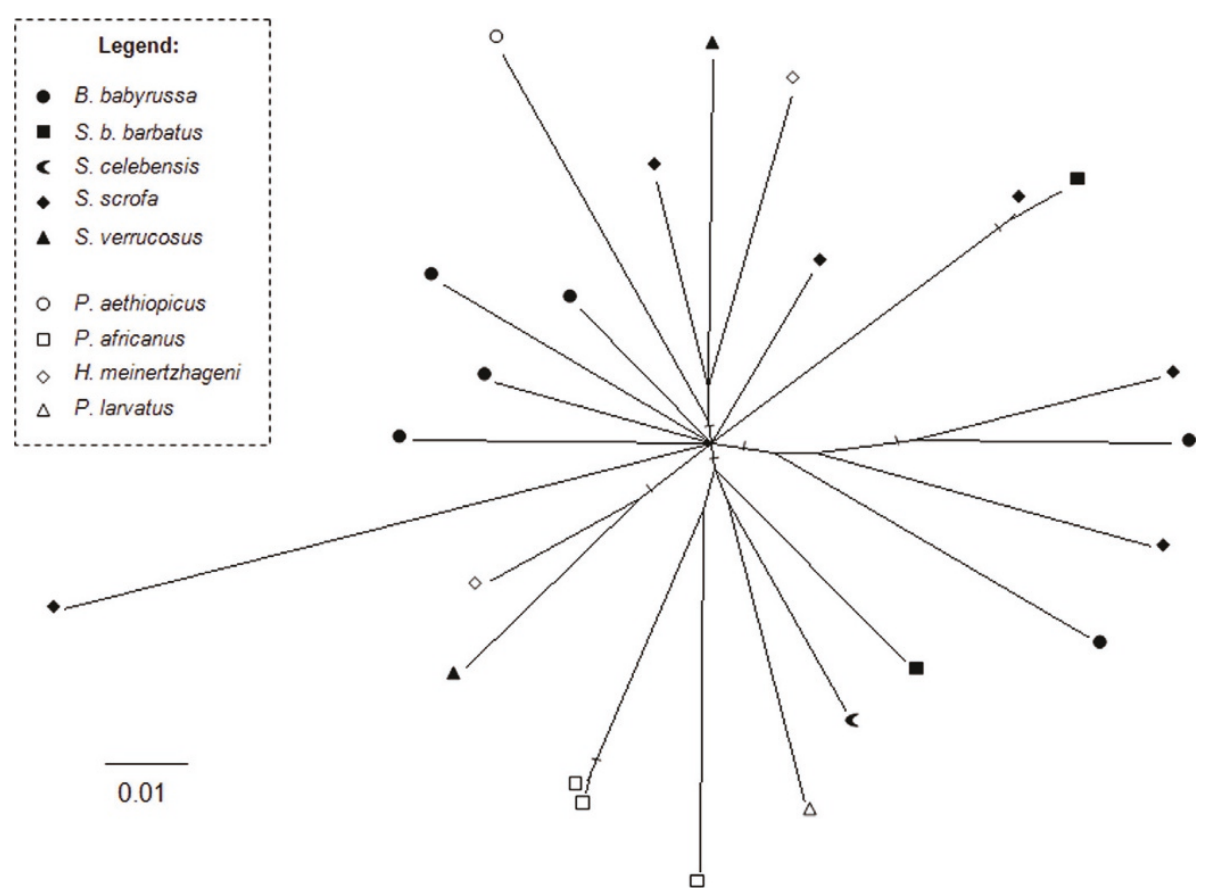

Figure 7 Unrooted Bayesian inference for env class E gene. Marked branches represent posterior probability values from 0.90-1.00 and posterior probability values for other branches ranged from 0.50-0.70 (not shown for clarity). Black symbols represent ERVs from the different Eurasian species and gray symbols represent ERVs from different African species (see legend for detail in coding). 
Table $2 d_{N} / d_{S}(\omega)$ ratios and likelihood ratio tests (LRT) for ERV genes in the family Suidae to test whether $\omega$ for internal branches are significantly less than 1

\begin{tabular}{|c|c|c|c|c|c|}
\hline \multicolumn{6}{|c|}{ Two-ratio model } \\
\hline Gene & $\begin{array}{c}\text { Internal } \\
\text { branches }(\omega)\end{array}$ & $\begin{array}{c}\text { Terminal } \\
\text { branches }(\omega)\end{array}$ & $\ell_{1}$ & $\ell_{0}$ & $\mathrm{LRT}^{1}$ \\
\hline gag & 0.34 & 0.78 & -3235.42 & -3253.27 & $38^{* *}$ \\
\hline pol & 0.25 & 0.56 & -5373.26 & -5405.94 & $65^{* *}$ \\
\hline env (class A) & 0.30 & 1.04 & -4271.15 & -4306.51 & $70^{* *}$ \\
\hline env (class B) & 0.22 & 1.47 & -2470.67 & -2515.98 & $90^{* *}$ \\
\hline \multicolumn{6}{|c|}{$\begin{array}{l}\ell=\log _{\mathrm{e}} \text {-likelihood } \\
{ }^{1} \mathrm{LRT}=\text { Likelihood ratio test, twice the difference of the log likelihoods }[2 \Delta \ell= \\
\left.2\left(\ell_{1}-\ell_{0}\right)\right] \\
{ }^{* *} \text { Extremely significant }\left(P<0.01 ; \chi_{1}{ }^{2}\right)\end{array}$} \\
\hline
\end{tabular}

Class A and B env phylogenies showed two likely subpopulations in African suids, one in Phacochoerus species, and another in Potamochoerus and Hylochoerus species more related to ERVs from Sus species, (Figures 3 and 4). Amplification of orthologous sequences as well as full length proviral sequences would provide a better basis for comparison, but for reasons of practicality and convenience paralogous sequences amplified by PCR have been routinely used to determine the evolutionary relationship of ERVs in different host species [41-43].

Recombination was apparent in several $\gamma 1$ and $\gamma 2$ sequences, presumably as a result of co-packaging of different RNAs in the same viral particle. In Sus scrofa, recombination between highly variable env sequences is easier to detect than for gag and pol. Moreover, selection would be also more likely to be operating on env than on the other genes because the viral envelope must evolve to escape immune detection, thus making recombination a likely source of adaptative phenotypes. ERVs from African species showed more evidence of recombination than ERVs from Eurasian species, although this apparent difference may be an artifact of the higher differentiation (longer branch lengths) for pol and for class A and B env in African species. Furthermore, the RDP 3 software identified a higher number of recombinants than the PHINNet algorithm for $\gamma 1$ genes, a fact also observed in HIV by Lamers et al. (2009) and attributed to the molecular models implemented in some RDP 3 methods, to the number of sequences in each analysis and to variation within each subpopulation altering the number of identified recombinants [44]. This might be why the $\gamma 1 \mathrm{pol}$ sequence from the draft pig genome was identified as recombinant by RDP 3 and non-recombinant by the PHINNet algorithm. These discordances obviously result from the different methods of analyses, pointing to the need of improving their consistency and power especially in detecting recombinants between ERV sequences. This improvement is also important to better characterize the inter subpopulation recombinants observed in this study and confirm that they represent genuine recombinant sequences.

We also found evidence for past purifying selection on all $\gamma 1$ genes. This suggested that retroviruses that remained functional were initially selected and that transposition by reinfection was relevant, while loss of function would have likely resulted in neutral evolution within the host genome $\omega \approx 1$. Furthermore, the rarity of stop codons and/or indels also suggested that complementation in trans and retrotransposition in cis were not relevant mechanisms of transposition $[17,18]$.

\section{Evolutionary comparisons of $\gamma 1$ and $\gamma 2$ env genes}

In contrast to the $\gamma 1$ phylogeny, $\gamma 2$ ERV env gene showed a bush-like unresolved tree, (Figure 7) confirmed by the likelihood-mapping, either resulting from inadequate sampling or data (soft polytomy) or reflecting the actual evolutionary history of $\gamma 2$ ERVs in the Suidae (hard polytomy) [45-47]. The bush-like pattern might reflect an incomplete sampling because env from ten other suid species is missing in the phylogeny. However, the $\gamma 1$ ERV env phylogenies (Figures 3 and 4) using fewer species showed a better resolved tree with species from Eurasia grouping apart from African suids, suggesting that the number of viral sequences samples from each suid host would not be an explanation for the poor resolution of $\gamma 2 \mathrm{ERV}$ env phylogeny.

An unresolved phylogeny, like that observed for $\gamma 2$ ERVs, may also result from data saturation, but the env sequences showed very low saturation. Although we cannot rule out the possibility that the $\gamma 2$ star-like phylogeny resulted from inadequate sampling, this seems unlikely as samples used here were available from ERVs from three different suid lineages observed by Nascimento [22,23]. In this case, a hard polytomy seems more plausible. Although Vandamme [45] emphasised the difficulty of proving the existence of hard polytomies in real life, Poe and Chubb [48], Barth et al. [46], Willerslev et al. [49] and several others [47,50] have observed some apparent examples in different taxa, including birds, mammals, plants and protozoa, and this pattern was also observed for human ERVs [19].

This hard polytomy may then represent a rapid radiation from a single common ancestor giving rise to multiple distinct retroviral lineages almost at the same time $[19,45]$. These ERVs have subsequently become inactivated resulting in the loss of the last active lineage (also suggested by the presence of several stop codons), following a long period of inactivity leading to the star shaped phylogeny [19].

The reason why distinct evolutionary histories are observed for $\gamma 1$ and $\gamma 2$ ERVs is not known. It would be reasonable to attribute it to the evolution of the host 
defensive mechanisms. However, Katzourakis et al. [19] by modelling ERV evolutionary dynamics demonstrated that such a star shaped phylogeny can be generated by a "null model" in which all parameters are set constant through out time.

\section{Conclusions}

The different lineages of $\gamma 1$ ERVs in the Suidae are generally congruent with the host phylogeny. This implies that horizontal transmission across these very different host lineages has not occurred. Moreover, the frequent evolutionary occurrence of viral recombination may be relevant to risk analysis of xenotransplantation, especially since viral recombination will favour adaptation and confer capacity for maintaining ongoing infection of somatic and germ cells. This highlights the importance of improving methods to prevent PERVs from crossing the species barriers. Finally, the evolution of $\gamma 2$ ERVs confirms their lack of risk in xenotransplantation because the star phylogeny suggests loss of the last active element which is also confirmed by the presence of numerous stop codons.

\section{Methods}

\section{Sample collection, primer design and PCR assays}

DNA samples of 12 animals from 10 species and one subspecies of Suidae (Table 1) were analyzed for the presence of ERVs. Oligonucleotide primers targeting conserved regions of the $\gamma 1 \mathrm{gag}$, pro/pol and env (classes A, B and C) genes and $\gamma 2$ env (class E; Table 3) were

Table 3 Oligonucleotide primer used to amplify and to sequence partial ERV genes

\begin{tabular}{|c|c|c|}
\hline Primer name & Primer sequences $\left(5^{\prime} \rightarrow 3^{\prime}\right)$ & Expected size (bp) \\
\hline \multirow[t]{2}{*}{ gag } & CTGTTGTTGAAGCGAAAG & 1,080 \\
\hline & TACCTTCAGCCGTGTTG & \\
\hline \multirow[t]{2}{*}{ pol } & ACCCGCTAACCAAAGA & 1,578 \\
\hline & TGTCTGACCCGATTACC & \\
\hline \multirow[t]{2}{*}{ env-A } & СCCGAACTCCCATAАACC & 1,720 \\
\hline & AAGGCCCAACTGTAAGTAACA* & \\
\hline \multirow[t]{2}{*}{ env-B } & CTGCGGCCTGACATAAC & 1,060 \\
\hline & AAGGCCCAACTGTAAGTAACA* & \\
\hline \multirow[t]{2}{*}{ env-C } & GAACCTGGTGGCCTGATCTAT & 1,524 \\
\hline & GGCCCAACTGTGAGTAACA & \\
\hline \multirow[t]{2}{*}{ env-E } & АССТСTTTGCCTGACAATACA & 1,320 \\
\hline & TACAAGGCAGGGAACAAGTAG & \\
\hline pol-intF ${ }^{1}$ & CAGACATACCGCTGACTG & $\mathrm{n} / \mathrm{a}$ \\
\hline pol-intR ${ }^{1}$ & TGTACTGTCATCCGGGTTCTG & $\mathrm{n} / \mathrm{a}$ \\
\hline env-A-intF ${ }^{1}$ & TGGTATGTCTTGGGGAAT & $\mathrm{n} / \mathrm{a}$ \\
\hline
\end{tabular}

designed using DNA sequences from Sus scrofa as input for OLIGO (version 6.8; Molecular Biology Insights). These specific primers were designed aiming to amplify from the other Suidae species similar sequences to Sus scrofa and increase the chances to amplify sequences in the same genomic loci. Products were PCR amplified with an initial denaturation at $94^{\circ} \mathrm{C}$ for $30 \mathrm{sec}$ followed by 35 cycles of denaturation at $94^{\circ} \mathrm{C}$ for $30 \mathrm{sec}$, annealing at $55^{\circ} \mathrm{C}$ or $58^{\circ} \mathrm{C}$ for $60 \mathrm{sec}$ (for gag and other genes respectively), extension at $72^{\circ} \mathrm{C}$ for $90 \mathrm{sec}$, and a final extension of $72^{\circ} \mathrm{C}$ for $5 \mathrm{~min}$. Amplicons were electrophoresed in a 1.5\% agarose gel and fragments of expected size (Table 3) were purified following gel band excision using UltraClean ${ }^{\mathrm{TM}}$ Gel Spin DNA Purification kit (Mo Bio, Australia).

\section{Cloning and sequencing}

Purified PCR products were cloned using the TOPO TA cloning ${ }^{\circledR}$ kit (Invitrogen, Australia) and DNA from plasmids containing inserts was subsequently extracted with UltraClean $^{\mathrm{TM}}$ Mini Plasmid Prep kit (Mo Bio). For each specimen, approximately five purified plasmids were sent to the Australian Genome Research Facility Ltd (AGRF; Brisbane, Australia) for DNA sequencing with primers listed in Table 3. Electropherograms were checked using BIOEDIT (version 7.0.9.0) [51].

\section{Data mining}

DNA sequences were blasted using the blastn option (http://blast.ncbi.nlm.nih.gov/Blast.cgi) to confirm that ERV products had been amplified and to identify all previously reported PERV sequences. The pre-ensembl Sus scrofa genome database (http://pre.ensembl.org/ index.html; version Sscrofa8) was searched using the blat option for gag, pro/pol and env, with best matching sequences being also included in alignments. Only sequences with $100 \%$ coverage were used in subsequent analyses. GenBank sequences were from genomic PERV sequences from pigs of various breeds, porcine cell lines, infected human primary cells and transcripts from virus particles released from porcine cell lines. The majority of GenBank sequences do not contain information on genomic position and we could not take this information into account. Sequences from the Sus scrofa genome were also not representative of the numerous PERV loci already described. This was because the Sscrofa8 version of the pig genome was not finished and because we have narrowed our search to sequences with $100 \%$ coverage.

\section{Alignment and genetic distance estimations}

All novel and available PERV sequences from GenBank and from the draft pig genome were aligned with MUSCLE (version 3.6) [52], with default options and were 
selection) was compared with the alternative hypothesis of $\omega<1$ (purifying selection). Significance was tested by fixing $\omega$ at 1 for internal branches and performing a likelihood ratio test (LRT), where twice the difference of the log-likelihoods was compared to critical values of the $\chi^{2}$ distribution for 1 degree of freedom [30].

For $\gamma 2$ env gene, we have compared a single $\omega$ for the entire tree (the "one-ratio" model) to a "free-ratio" model (a separate $\omega$ ratio for each branch of the phylogeny) [18]. The significance of differences between "oneratio" and "free-ratio" models was assessed by a LRT for 34 degrees of freedom [30].

Outgroups were removed from the trees before performing these calculations.

\section{GenBank accession numbers}

env class E: [GQ906159-GQ906198]; env class A: [GQ906199-GQ906216]; env class B: [GQ906217GQ906249]; env class C: [GQ906250-GQ906271]; gag: [GQ906272-GQ906309]; pol: [GQ906310-GQ906342] for sequences generated in this study. For sequences retrieved from GenBank see additional file 1.

Multiple sequence alignments are provided as additional files 2, 3, 4, 5, 6 and 7 .

\section{Additional material}

Additional file 1: GenBank accession numbers for sequences included in this study. List of GenBank sequences used in phylogenetic analyses

Additional file 2: gag alignment. gag alignment of sequences generated in this study, sequences from GenBank and the draft pig genome

Additional file 3: pol alignment. pol alignment of sequences generated in this study, sequences from GenBank and the draft pig genome

Additional file 4: env A alignment. env A alignment of sequences generated in this study, sequences from GenBank and the draft pig genome

Additional file 5: env B alignment. env B alignment of sequences generated in this study, sequences from GenBank and the draft pig genome

Additional file 6: env C alignment. env $C$ alignment of sequences generated in this study, sequences from GenBank and the draft pig genome

Additional file 7: env $\mathrm{E}$ alignment. env $\mathrm{E}$ alignment of sequences generated in this study, sequences from GenBank and the draft pig genome

\section{Acknowledgements and Funding}

F. F. Nascimento was a grantee of EIPRS and Stock and Meat Industries Grant-in Aid (Australia), and Boehringer Ingelheim Fonds (Germany). M. Charleston gratefully acknowledges funding from the Australian Research Council (grant number DP1094891). We are grateful for $\mathrm{H}$. Klingel (Ugandan Institute of Ecology, Uganda, and University of Braunschweig, Germany) and to E. Randi (Istituto Superiore per la Protezione e la Ricerca Ambientale, Italy) for providing the DNA samples of the forest hog and the desert warthog respectively. We are thankful to $\mathrm{H}$. Seuánez (Instituto Nacional de Câncer,
Brazil) and G. Larson (Durham University, United Kingdom) for reviewing earlier drafts of this manuscript.

\section{Author details}

${ }^{1}$ Faculty of Veterinary Science, The University of Sydney, NSW 2006, Australia. ${ }^{2}$ School of Information Technologies and Centre for Mathematical Biology, The University of Sydney, NSW 2006, Australia. ${ }^{3}$ Division of Biology, Imperial College London, Silwood Park, Ascot, Berkshire SL5 7PY, UK. ${ }^{4}$ Veterinary Health Research Pty Ltd. Armidale, NSW 2350, Australia. ${ }^{5}$ Instituto de Microbiologia Paulo de Góes, Universidade Federal do Rio de Janeiro, Avenida Carlos Chagas Filho, 373, Rio de Janeiro 21941-902, Brazil.

\section{Authors' contributions}

FFN generated data, carried out molecular, evolutionary and statistical analyses and drafted the manuscript. JG participated in the design of the study and provided assistance with interpretation of results. MC provided special input in phylogenetic and co-phylogenetic analyses. MT provided special input into the analysis and interpretation of retroviral data. SL collected and provided genomic DNA samples from most suid host species. CM initiated and oversaw project, provided assistance with interpretation of results and redrafting of manuscript. All authors read and approved the final manuscript.

Received: 23 November 2010 Accepted: 24 May 2011

Published: 24 May 2011

\section{References}

1. Gifford R, Tristem M: The evolution, distribution and diversity of endogenous retroviruses. Virus Genes 2003, 26:291-315.

2. Herniou E, Martin J, Miller K, Cook J, Wilkinson M, Tristem M: Retroviral diversity and distribution in vertebrates. J Virol 1998, 72:5955-5966.

3. Weiss RA: The discovery of endogenous retroviruses. Retrovirology 2006, 3:67.

4. Biemont C, Vieira C: Genetics: junk DNA as an evolutionary force. Nature 2006, 443:521-524

5. Patience C, Switzer WM, Takeuchi Y, Griffiths DJ, Goward ME, Heneine W, Stoye JP, Weiss RA: Multiple groups of novel retroviral genomes in pigs and related species. J Virol 2001, 75:2771-2775.

6. Klymiuk N, Müller M, Brem G, Aigner B: Characterization of porcine endogenous retrovirus gamma pro-pol nucleotide sequences. J Virol 2002, 76:11738-11743.

7. Martin U, Kiessig V, Blusch JH, Haverich A, von der Helm K, Herden T, Steinhoff $G$ : Expression of pig endogenous retrovirus by primary porcine endothelial cells and infection of human cells. Lancet 1998, 352:692-694.

8. Takeuchi Y, Patience C, Magre S, Weiss RA, Banerjee PT, Le Tissier P, Stoye JP: Host range and interference studies of three classes of pig endogenous retrovirus. J Virol 1998, 72:9986-9991.

9. Patience C, Takeuchi Y, Weiss RA: Infection of human cells by an endogenous retrovirus of pigs. Nat Med 1997, 3:282-286.

10. Stoye JP, Coffin JM: The dangers of xenotransplantation. Nat Med 1995, $1: 1100$.

11. Platt JL: Xenotransplantation. New risks, new gains. Nature 2000, 407:29-30, 27.

12. Boneva RS, Folks TM: Xenotransplantation and risks of zoonotic infections. Ann Med 2004, 36:504-517.

13. Smetanka C, Cooper DK: The ethics debate in relation to xenotransplantation. Rev Sci Tech 2005, 24:335-342.

14. Mang R, Maas J, Chen X, Goudsmit J, van Der Kuyl AC: Identification of a novel type $C$ porcine endogenous retrovirus: evidence that copy number of endogenous retroviruses increases during host inbreeding. J Gen Virol 2001, 82:1829-1834.

15. Klymiuk N, Muller M, Brem G, Aigner B: Phylogeny, recombination and expression of porcine endogenous retrovirus gamma2 nucleotide sequences. J Gen Virol 2006, 87:977-986.

16. Niebert M, Tönjes RR: Evolutionary spread and recombination of porcine endogenous retroviruses in the Suiformes. J Virol 2005, 79:649-654.

17. Belshaw R, Katzourakis A, Paces J, Burt A, Tristem M: High copy number in human endogenous retrovirus families is associated with copying mechanisms in addition to reinfection. Mol Biol Evol 2005, 22:814-817.

18. Belshaw R, Pereira V, Katzourakis A, Talbot G, Paces J, Burt A, Tristem M: Long-term reinfection of the human genome by endogenous retroviruses. Proc Natl Acad Sci USA 2004, 101:4894-4899. 
19. Katzourakis A, Rambaut A, Pybus OG: The evolutionary dynamics of endogenous retroviruses. Trends Microbiol 2005, 13:463-468.

20. Finnegan DJ: Transposable elements. Curr Opin Genet Dev 1992, 2:861-867.

21. Jern P, Coffin JM: Effects of retroviruses on host genome function. Annu Rev Genet 2008, 42:709-732

22. Nascimento FF: Evolution of the family Suidae and their endogenous retroviruses. PhD The University of Sydney; 2009

23. Gongora J, Cuddahee RE, Nascimento FF, Palgrave CJ, Lowden S, Ho SYW, Simond D, Damayanti CS, White DJ, Tay WT, Randi E, Klingel H, RodriguesZarate CJ, Allen K, Moran C, Larson G: Rethinking the evolution of subSaharan African suids (Suidae, Artiodactyla). Zool Scr 2011.

24. Martin DP, Williamson C, Posada D: RDP2: recombination detection and analysis from sequence alignments. Bioinformatics 2005, 21:260-262.

25. Salemi M, Gray RR, Goodenow MM: An exploratory algorithm to identify intra-host recombinant viral sequences. Mol Phylogenet Evol 2008, 49:618-628.

26. Xia X, Xie Z, Salemi M, Chen L, Wang Y: An index of substitution saturation and its application. Mol Phylogenet Evol 2003, 26:1-7.

27. Xia X: Assessing substitution saturation with DAMBE. In The phylogenetic handbook: a practical approach to phylogenetic analysis and hypothesis testing. 2 edition. Edited by: Salemi M, Vandamme A-M, Lemey P. Cambridge: Cambridge University Press; 2009:615-623.

28. Strimmer K, von Haeseler A: Likelihood-mapping: a simple method to visualize phylogenetic content of a sequence alignment. Proc Natl Acad Sci USA 1997, 94:6815-6819.

29. Yang Z, Bielawski JP: Statistical methods for detecting molecular adaptation. Trends Ecol Evol 2000, 15:496-503.

30. Yang Z: Likelihood ratio tests for detecting positive selection and application to primate lysozyme evolution. Mol Biol Evol 1998, 15:568-573.

31. Nascimento FF, Gongora J, Tristem M, Lowden S, Moran C: Distinctive differences in long terminal repeat sequences between gamma1 endogenous retroviruses of African and Eurasian suid species. Infect Genet Evol 2011, 11:686-693.

32. Harris JM, White TD: Evolution of the Plio-Pleistocene African Suidae. Trans Am Phil Soc 1979, 69:1-128.

33. White TD, Harris JM: Suid evolution and correlation of African hominid localities. Science 1977, 198:13-21.

34. Pickford M: Old world suoid systematics, phylogeny, biogeography and biostratigraphy. Paleontologia i Evolució 1993, 26-27:237-269.

35. Jeffroy O, Brinkmann H, Delsuc F, Philippe H: Phylogenomics: the beginning of incongruence? Trends Genet 2006, 22:225-231.

36. Rannala B, Yang Z: Phylogenetic inference using whole genomes. Annu Rev Genomics Hum Genet 2008, 9:217-231.

37. Degnan JH, Rosenberg NA: Gene tree discordance, phylogenetic inference and the multispecies coalescent. Trends Ecol Evol 2009, 24:332-340.

38. Llorens C, Fares MA, Moya A: Relationships of gag-pol diversity between Ty3/Gypsy and Retroviridae LTR retroelements and the three kings hypothesis. BMC Evol Biol 2008, 8:276

39. Howard TM, Sheng Z, Wang M, Wu Y, Rasheed S: Molecular and phylogenetic analyses of a new amphotropic murine leukemia virus (MuLV-1313). Virol J 2006, 3:101.

40. Auerbach MR, Shu C, Kaplan A, Singh IR: Functional characterization of a portion of the Moloney murine leukemia virus gag gene by genetic footprinting. Proc Natl Acad Sci USA 2003, 100:11678-11683.

41. Jaratlerdsiri W, Rodriguez-Zarate CJ, Isberg SR, Damayanti CS, Miles LG, Chansue N, Moran C, Melville L, Gongora J: Distribution of endogenous retroviruses in crocodilians. J Virol 2009, 83:10305-10308.

42. Xiao R, Park K, Lee $H$, Kim J, Park C: Identification and classification of endogenous retroviruses in cattle. J Virol 2008, 82:582-587.

43. Tristem $M$, Kabat $P$, Lieberman $L$, Linde $S$, Karpas A, Hill F: Characterization of a novel murine leukemia virus-related subgroup within mammals. J Virol 1996, 70:8241-8246.

44. Lamers SL, Salemi M, Galligan DC, de Oliveira T, Fogel GB, Granier SC, Zhao L, Brown JN, Morris A, Masliah E, McGrath MS: Extensive HIV-1 intrahost recombination is common in tissues with abnormal histopathology. PLoS One 2009, 4:e5065.

45. Vandamme AM: Basic concepts of molecular evolution. In The phylogenetic handbook: a practical approach to phylogenetic analysis and hypothesis testing.. 2 edition. Edited by: Salemi M, Vandamme A-M, Lemey P. Cambridge: Cambridge University Press; 2009:3-29.

46. Barth D, Przybos E, Fokin SI, Schlegel M, Berendonk TU: Cytochrome $b$ sequence data suggest rapid speciation within the Paramecium aurelia species complex. Mol Phylogenet Evol 2008, 49:669-673.

47. Whitfield JB, Lockhart PJ: Deciphering ancient rapid radiations. Trends Ecol Evol 2007, 22:258-265.

48. Poe $\mathrm{S}$, Chubb AL: Birds in a bush: five genes indicate explosive evolution of avian orders. Evolution 2004, 58:404-415.

49. Willerslev E, Gilbert MT, Binladen J, Ho SY, Campos PF, Ratan A, Tomsho LP, da Fonseca RR, Sher A, Kuznetsova TV, Nowak-Kemp M, Roth TL, Miller W, Schuster SC: Analysis of complete mitochondrial genomes from extinct and extant rhinoceroses reveals lack of phylogenetic resolution. BMC Evol Biol 2009, 9:95

50. Rokas A, Carroll SB: Bushes in the tree of life. PLoS Biol 2006, 4:e352.

51. Hall TA: BioEdit: a user-friendly biological sequence alignment editor and analysis program for Windows 95/98/NT. Nucleic Acids Symp Ser 1999, 41:95-98.

52. Edgar RC: MUSCLE: multiple sequence alignment with high accuracy and high throughput. Nucleic Acids Res 2004, 32:1792-1797.

53. Yang Z: Computational molecular evolution Oxford: Oxford University Press; 2006.

54. Galtier N, Gouy M, Gautier C: SEAVIEW and PHYLO_WIN: two graphic tools for sequence alignment and molecular phylogeny. Comput Appl Biosci 1996, 12:543-548.

55. Tamura K, Dudley J, Nei M, Kumar S: MEGA4: Molecular Evolutionary Genetics Analysis (MEGA) software version 4.0. Mol Biol Evol 2007, 24:1596-1599.

56. Tamura K, Kumar S: Evolutionary distance estimation under heterogeneous substitution pattern among lineages. Mol Biol Evol 2002, 19:1727-1736.

57. Hudson RR, Boos DD, Kaplan NL: A statistical test for detecting geographic subdivision. Mol Biol Evol 1992, 9:138-151.

58. Achaz G, Palmer S, Kearney M, Maldarelli F, Mellors JW, Coffin JM, Wakeley J: A robust measure of HIV-1 population turnover within chronically infected individuals. Mol Biol Evol 2004, 21:1902-1912.

59. Kosiol C, Bofkin L, Whelan S: Phylogenetics by likelihood: evolutionary modeling as a tool for understanding the genome. J Biomed Inform 2006, 39:51-61.

60. Jermiin $L S$, Jayaswal $V$, Ababneh F, Robinson J: Phylogenetic model evaluation. Methods Mol Biol 2008, 452:331-364.

61. Martin D, Rybicki E: RDP: detection of recombination amongst aligned sequences. Bioinformatics 2000, 16:562-563.

62. Sawyer S: Statistical tests for detecting gene conversion. Mol Biol Evol 1989, 6:526-538.

63. Padidam M, Sawyer S, Fauquet CM: Possible emergence of new geminiviruses by frequent recombination. Virology 1999, 265:218-225.

64. Smith JM: Analyzing the mosaic structure of genes. J Mol Evol 1992, 34:126-129.

65. Posada D, Crandall KA: Evaluation of methods for detecting recombination from DNA sequences: computer simulations. Proc Nat Acad Sci USA 2001, 98:13757-13762.

66. Gibbs MJ, Armstrong JS, Gibbs AJ: Sister-scanning: a Monte Carlo procedure for assessing signals in recombinant sequences. Bioinformatics 2000, 16:573-582

67. Boni MF, Posada D, Feldman MW: An exact nonparametric method for inferring mosaic structure in sequence triplets. Genetics 2007, 176:1035-1047.

68. Huelsenbeck JP, Ronquist F: MRBAYES: Bayesian inference of phylogenetic trees. Bioinformatics 2001, 17:754-755

69. Ronquist F, Huelsenbeck JP: MrBayes 3: Bayesian phylogenetic inference under mixed models. Bioinformatics 2003, 19:1572-1574.

70. Keane TM, Creevey CJ, Pentony MM, Naughton TJ, McLnerney JO: Assessment of methods for amino acid matrix selection and their use on empirical data shows that ad hoc assumptions for choice of matrix are not justified. BMC Evol Biol 2006, 6:29.

71. Nylander JA, Ronquist F, Huelsenbeck JP, Nieves-Aldrey JL: Bayesian phylogenetic analysis of combined data. Syst Biol 2004, 53:47-67.

72. Tracer v1.4. [http://beast.bio.ed.ac.uk/Tracer]

73. Xia X, Xie Z, Salemi M, Chen L, Wang Y: An index of substitution saturation and its application. Mol Phylogenet Evol 2003, 26:1-7. 
74. Xia X, Xie Z: DAMBE: software package for data analysis in molecular biology and evolution. J Hered 2001, 92:371-373.

75. Schmidt HA, Strimmer K, Vingron M, von Haeseler A: TREE-PUZZLE: maximum likelihood phylogenetic analysis using quartets and parallel computing. Bioinformatics 2002, 18:502-504.

76. Schmidt $H A$, von Haeseler A: Phylogenetic inference using maximum likelihood methods. In The phylogenetic handbook : a practical approach to phylogenetic analysis and hypothesis testing.. 2 edition. Edited by: Salemi M, Vandamme A-M, Lemey P. Cambridge: Cambridge University Press; 2009:181-198.

77. Yang Z: PAML 4: phylogenetic analysis by maximum likelihood. Mol Biol Evol 2007, 24:1586-1591.

78. Hasegawa M, Kishino $\mathrm{H}$, Yano $\mathrm{T}$ : Dating of the human-ape splitting by a molecular clock of mitochondrial DNA. J Mol Evol 1985, 22:160-174.

79. Kimura M: A simple method for estimating evolutionary rates of base substitutions through comparative studies of nucleotide sequences. J Mol Evol 1980, 16:111-120.

80. Kass RE, Raftery AE: Bayes factors. J Am Stat Assoc 1995, 90:773-795.

doi:10.1186/1471-2148-11-139

Cite this article as: Nascimento et al:: Evolution of endogenous

retroviruses in the Suidae: evidence for different viral subpopulations in

African and Eurasian host species. BMC Evolutionary Biology 2011 11:139.

\section{Submit your next manuscript to BioMed Central} and take full advantage of:

- Convenient online submission

- Thorough peer review

- No space constraints or color figure charges

- Immediate publication on acceptance

- Inclusion in PubMed, CAS, Scopus and Google Scholar

- Research which is freely available for redistribution

Submit your manuscript at www.biomedcentral.com/submit 